\title{
Evaluación cualitativa, comprensiva y comparativa del funcionamiento del Sistema Nacional De Planificación de Haití
}

\author{
Willy François
}

Universidad de Chile

\section{Resumen}

Se analizó la estructura y gestión del sistema nacional de planificación de Haití, a través de un modelo teórico elaborado por el Instituto Latinoamericano y del Caribe de Planificación Económica Social (ILPES). El análisis se basó en una comparación a nivel estructural de los sistemas de planificación de Haití y de República Dominica, y en una encuesta realizada en el Ministerio de Planificación y de la Cooperación Externa (MPCE) de Haití, con el fin de identificar los nodos críticos de gestión. Se pudo constatar que el sistema de planificación de Haití cuenta con casi todos los componentes que necesita un sistema de planificación, sin embargo se destacan algunas fallas en la estructura de planificación. Adicionalmente, se identificaron algunos nodos críticos de estructura, producto de la ausencia de leyes que constituyan un marco legal para definir las funciones, los roles, los atributos y la gestión. Del mismo modo se destacó una ruptura entre el nivel estratégico y los niveles programáticos y operativos

Palabras clave: Gestión pública, gestión de estructuras administrativas, enfoque sistémico.

Qualitative, comprehensive and comparative evaluation of the functioning of Haiti National Planning System

\begin{abstract}
The structure and management of the national planning system in Haiti was analyzed using a theoretical model developed by the Instituto Latinoamericano y del Caribe de Planificación Económica Social (ILPES). The analysis was based on a comparison among planning systems structural level of Haiti and República Dominicana, and on a survey administrated to the Ministry of Planning and External Cooperation (MPCE) of Haiti. The purpose was to identify the management critical nodes. We founded that the Haiti planning system had almost all the components that a planning system required, however it highlighted some faults in the structure planning. Additionally, some structure critical nodes were identified, because of the absence of laws that constitute a legal framework for defining the functions, roles, attributes and management. A break among the strategic level and programmatic and operational levels was highlighted
\end{abstract}

Keywords: Public management, administrative structures management, systemic approach.

*Dirección de correspondencia [Correspondence

address]: Willy François, Universidad de Chile

E-mail: franly001@gmail.com 


\section{Introducción}

La planificación económica y social surgió en América Latina alrededor de los años 60. Después de un periodo de prueba y error, basándose sobre las recomendaciones de la ILPES, muchos países de la región desarrollaron un sistema de planificación que les han permitido transformar sus estructuras económicas para dar mayor respuesta a las necesidades de la población. La Republica de Haití, pese a que tuvo una estructura de planificación desde los años 70 y un Ministerio desde el año 1989, no ha logrado desarrollarse y responder a las necesidades de su población.

La "planificación para el desarrollo" tiene como premisa inicial buscar transformaciones cualitativas concurrentemente con incrementos cuantitativos, a través de un "proceso dirigido a orientar o impulsar el cambio social, en el cual los diferentes actores sociales, interviniendo de manera sistémica en el presente para construir el futuro, garantizan el logro del desarrollo deseado y posible, protegiendo las opciones para las generaciones futuras" (Saavedra Guzman, 2003: 49). Para la consecución de estos objetivos, esta noción se basa en los siguientes seis aspectos centrales: primero, el proceso de planificación se da como resultado de la participación de los diferentes actores sociales, por lo que su validación es eminentemente social; segundo, esta participación es generadora de consenso, en donde se reconoce la responsabilidad que tiene cada actor en este proceso; tercero, el pensamiento a futuro implica necesariamente mejorar la gestión actual, generando cambios y resultados; cuarto, adoptando una visión holística del proceso, articulando e integrando procesos, subprocesos y componentes requeridos para el desarrollo; quinto, la capacidad dinámica y anticipativa de los actores sociales y; finalmente, la interactividad del proceso, a través de instancias de retroalimentación y ajuste.

Los elementos que estructuran la planificación del desarrollo, se pueden articular gráficamente de la siguiente manera:

Como en todo sistema funcional, la "planificación para el desarrollo" es una acción compleja que necesita una acción coordinada y sistémica dado que resulta de las intervenciones de distintos actores interviniendo en distintos ámbitos. Es necesario, por lo tanto, la elaboración de un marco conceptual que permita un diseño estructural de todo el sistema, orientado hacia la eficiencia, la eficacia y la calidad de las acciones llevadas a cabo.

Por ello, partiendo del modelo del ILPES, sobre el manejo de estructura del sistema nacional de planificación y de gestión, analizaremos el sistema de
Figura 1: Interrelación de los elementos de la planificación del desarrollo

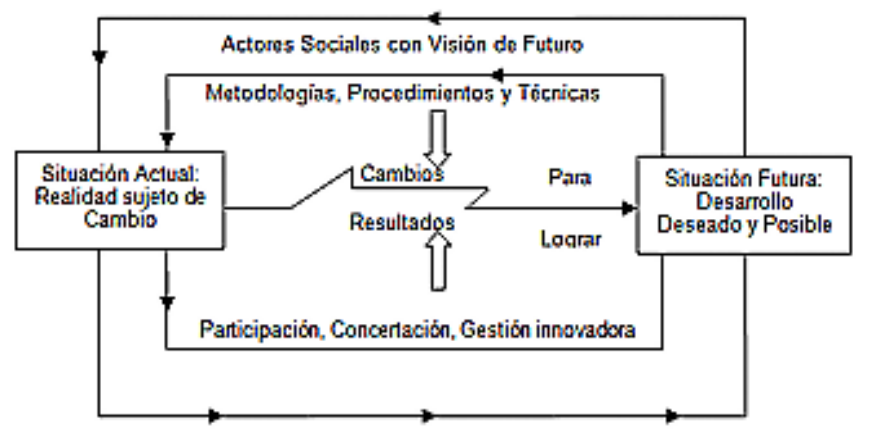

Fuente: Saavedra Guzman (2003)

planificación haitiano, buscando encontrar las principales razones institucionales y estructurales que impiden que el sistema de planificación pueda cumplir con las metas fijadas. El estudio de base es un análisis comparativo entre el sistema de planificación haitiano y otros sistemas de la región que han tenido éxito. Se plantea así la siguiente pregunta de investigación: ¿La estructura del sistema de planificación y la forma de gestión permiten desarrollar las funciones pertinentes para la planificación del desarrollo?

De esta manera, el objetivo de la presente investigación es analizar el sistema de planificación de Haití desde la perspectiva de la estructura y de la gestión a la luz de un modelo teórico con el fin de ver si en dicho sistema se pueden realizar actividades de mejora.

\section{Manejo de estructuras}

La administración pública gestiona múltiples y complejos objetivos. El conjunto de los objetivos ministeriales componen el objetivo del Estado: entrega del valor público (Moore, 1998). Este objetivo es mucho más complejo que la suma de los objetivos institucionales por separado. La relación entre las instituciones del Estado y los ciudadanos no es una transacción proveedor-cliente, sino una relación en función de los objetivos complejos del Estado. Por ende, esta relación debe ser sinérgica.

La estructura administrativa de las organizaciones sociales ha sido tratada desde diversos prismas, pero tiene sus bases en la concepción de la especialización del trabajo y distribución del poder, autoridad y responsabilidad. En acuerdo con esto, (Weber, 1964) definió un tipo ideal de burocracia caracterizada principalmente por la jerarquía de autoridad y las reglas escritas. La burocracia intenta asegurar que las decisiones se tomen de acuerdo 
con criterios generales. Se establece que la experticia que adquieren los funcionarios, asegura un nivel general de competencia. Giddens (1994), por su parte, entiende la rutina burocrática y la autoridad que ejercen los funcionarios sobre nuestras vidas como el precio que se paga por la eficacia técnica de la organización burocrática.

En contraposición a la visión burocrática de Weber, Motta (1993) plantea una búsqueda de flexibilidad. Esta pasa por diferentes etapas, partiendo de la Perspectiva Sistémica y Dinámica, la Contingencial, Post Contingencial, la Perspectiva Práctica, hasta llegar, finalmente, a la propuesta de la Organización Atomizada.

\section{Manejo de la Estructura desde el En- foque Mintzberiano}

El enfoque basado en el modelo de Henry Mintzberg (1999), sostiene que las organizaciones están planteadas para captar y dirigir sistemas de flujos y para definir las interrelaciones entre sus distintas partes, entre las cuales se identifica:

- Núcleo de operaciones (N.O.): Son aquellas personas encargadas de la elaboración del producto o servicio principal de la organización, el que se entrega directamente a la comunidad. Desarrolla cuatro funciones principales: 1) aseguran los insumos para la producción, 2) los transforman en productos, 3) distribuyen los productos y 4) proveen apoyo directo a la entrada, transformación y salida de insumos y productos.

- Cumbre o Ápice estratégico: Corresponde a aquellas personas que asumen la responsabilidad por el desempeño general de la organización. Generalmente son el Director de la organización y todos sus gerentes de división o alto nivel, cuyos intereses son más bien globales. Su misión principal es la efectividad organizacional, así como también satisfacer las necesidades de los usuarios y el gobierno superior. Sus funciones principales son: 1) asignar recursos, emitir órdenes de trabajo, resolver conflictos y nombrar al personal de la organización, 2) administrar las condiciones fronterizas de la organización, informar a los actores influyentes, establecer contactos de alto nivel y negociar acuerdos y, 3) desarrollar la estrategia general de la organización, así como también generar corrientes de decisión tendientes a materializarla.

- Línea media: Es la cadena que recorre desde los altos directivos hasta los supervisores de con- tacto. Une la Cumbre Estratégica con el Nivel Operacional. Este eslabón depende del crecimiento de la organización y las necesidades de supervisión.

- La tecnoestructura: Son aquellas personas ajenas a la línea jerárquica, preocupadas de la normalización y estandarización de las tareas y trabajos. Sirven a la organización afectando el trabajo de otros. Sus funciones son: 1) la adaptación de la organización al medio (planificación estratégica), el control de gestión y los estudios sobre la carga, y 2) la organización o estandarización de normas.

- Staff de apoyo: Corresponden a personas y unidades especializadas en la entrega de servicios indirectos a la organización. Hablamos de unidades de asesoría legal, servicios generales, cafetería, seguridad, remuneraciones, central telefónica, etc.

- Ideología o cultura: Tanto dentro como fuera de la organización, corresponde al conjunto de valores y creencias que influyen sobre el esqueleto de la organización y la forma en que está dividido el trabajo para el manejo de insumos.

La predominancia, injerencia o tamaño relativo de cada uno de estos elementos generan diversos modelos y formas de configuración organizacional. Estos parámetros de diseño de la estructura se conjugan con diversos mecanismos coordinadores, los cuales, dependiendo de las necesidades y tipo de organización pueden ser: 1) Supervisión directa, 2) la Estandarización de procesos, 3) Estandarización de destrezas, 4) Estandarización de productos, 5) Adaptación mutua y, 6) Estandarización de reglas.

Figura 2: Estructura de las organizaciones según Minztberg

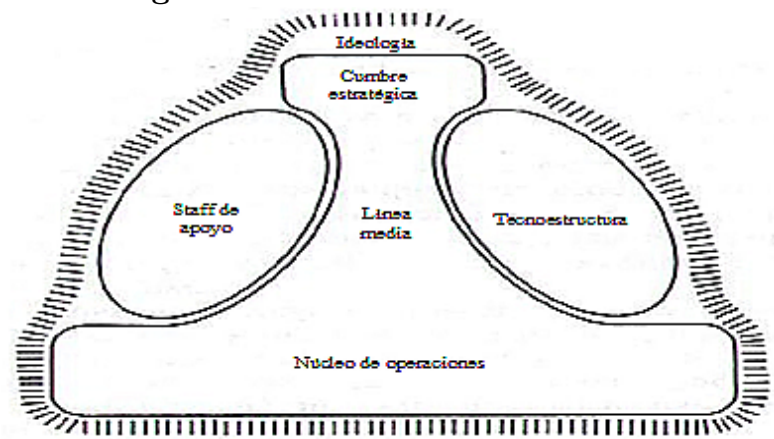

Fuente: Mintzberg (1999)

Hay que tener en cuenta las consideraciones de contexto que terminan de dar forma a la estructura: 1) la edad y el tamaño de la organización, 2) las características de los sistemas técnicos de producción, 
3) el grado de control externo de la organización (poder), y 4) el entorno económico e institucional de mercado u otras agencias del sector público.

De acuerdo con lo planteado en el enfoque post contingencial, las estructuras organizacionales deben ser flexibles y adaptables al entorno, estableciendo una interdependencia entre estrategia y estructura. Esto implica, entre otras cosas, que los análisis anticipatorios de las relaciones entre estrategia, estructura y proceso gerencial sean vitales para la modelación de estructuras adecuadas a los cambios en el medio ambiente (Pacheco, 2012). Si la inflexibilidad era el signo característico de los organismos públicos, hoy la complejidad del entorno lleva a pensar en buscar formas más adecuadas para establecer parámetros tanto en la evaluación como en el diseño de estos.

\section{Manejo de Estructuras desde el Enfo- que Sistémico}

Tanto la sociedad en su conjunto como las organizaciones pueden ser vistas analíticamente como sistemas abiertos. Un sistema abierto es aquel que tiene un contacto permanente y bidireccional con el exterior. El sistema recibe insumo del entorno, que luego procesa generando un producto. La retroalimentación se produce en la medida en que los nuevos insumos del sistema estuvieron afectados previamente con el output que el mismo produjo.

Katz y Kahn (1977) identifican cinco subsistemas que actúan en el interior de cada sistema:

- Subsistema de Adaptación: Gestiona las presiones externas de la organización. Permite adaptarse a los cambios y responder a nuevas demandas.

- Subsistema de Mantenimiento: Encargado de proveer la energía necesaria para mantener la estabilidad y capacidad de producción. Procura el mantenimiento del status quo.

- Subsistema Gerencial: Dirige y controla la acción. Resuelve conflictos en niveles de jerarquía, coordina y dirige a los otros subsistemas, y coordina los requerimientos externos y las necesidades organizacionales.

- Subsistema de Apoyo: proporciona una fuente de recursos que permiten generar productos. Este subsistema trabaja en el límite de la organización, manteniendo transacciones con el exterior, capturando apoyo social.

- Subsistema de Producción: Procesa la energía que ingresa al sistema y genera el producto que se envía al entorno. Es altamente dependiente de los demás subsistemas.

Las instituciones del Estado no son procesos aislados, sino partes interdependientes de un sistema. Reciben insumos como información, recursos financieros, normas, proyectos y estímulos provenientes de la ciudadanía o la esfera política; que procesan para transformarlos en resultados, que pueden ser bienes, servicios o incluso información. Estas salidas pueden ser los insumos de otra institución, configurando una red compleja de sistemas que persiguen objetivos en común.

\section{Dinámica interorganizacional}

La formulación de políticas públicas es un proceso complejo, en la medida en que vincula organizaciones con culturas organizacionales, estilos gerenciales y lógicas internas eventualmente distintas. La dinámica interoganizacional debe darse entre los distintos niveles, desde los estratégicos (ápice estratégico) hasta los operativos (núcleo operacional). La articulación entre organismos de un sistema lleva necesariamente a clarificar las funciones que asume cada una de las organizaciones.

Los mecanismos de coordinación a diferente escala permiten mejorar la toma de decisiones de las organizaciones involucradas. Mientras más plana es la estructura de cada uno de los organismos interrelacionados, el vínculo entre niveles decisionales (ápice estratégico) es mejor, ya que el flujo de información ascendente se vuelve más eficiente. En diferente sentido, el flujo descendente de información mejora y el nivel operativo de un órgano sabrá precisamente cómo debe ser la comunicación con un par en otra organización que comparte sus funciones, reduciendo el problema de cuarto oscuro planteado por (Crozier, 1969).

En cuanto a la vinculación a nivel sistémico, cabe destacar la importancia de la información entre los organismos que forman parte de un sistema, ya que al concebir que cada sistema se articula en torno a un órgano rector, este se nutre de las funciones específicas que cada uno de los organismos y subsistemas relacionados, le dotan para el correcto funcionamiento del mismo. Al aplicar esta vinculación al Sistema de Planificación se puede apreciar el siguiente esquema:

La organización político-administrativa de cada país depende de la forma de Estado adoptado, por tanto, es posible encontrar diferencias en la organización territorial federal y unitaria. A grandes rasgos, la vinculación entre organismos de distinto nivel territorial puede ser de dos naturalezas: bajo 
Figura 3: Vinculación interorganizacional a niveles decisionales/estratégicos de organismos de un sistema

\section{Niveles decisionales Organismo A Organismo B}

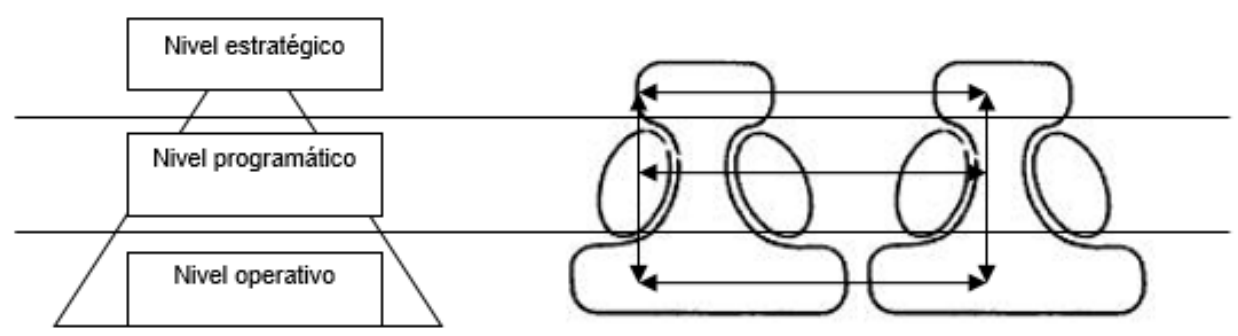

Fuente: Juan Francisco Pacheco

Figura 4: Vinculación sistémica aplicada al Sistema de Planificación

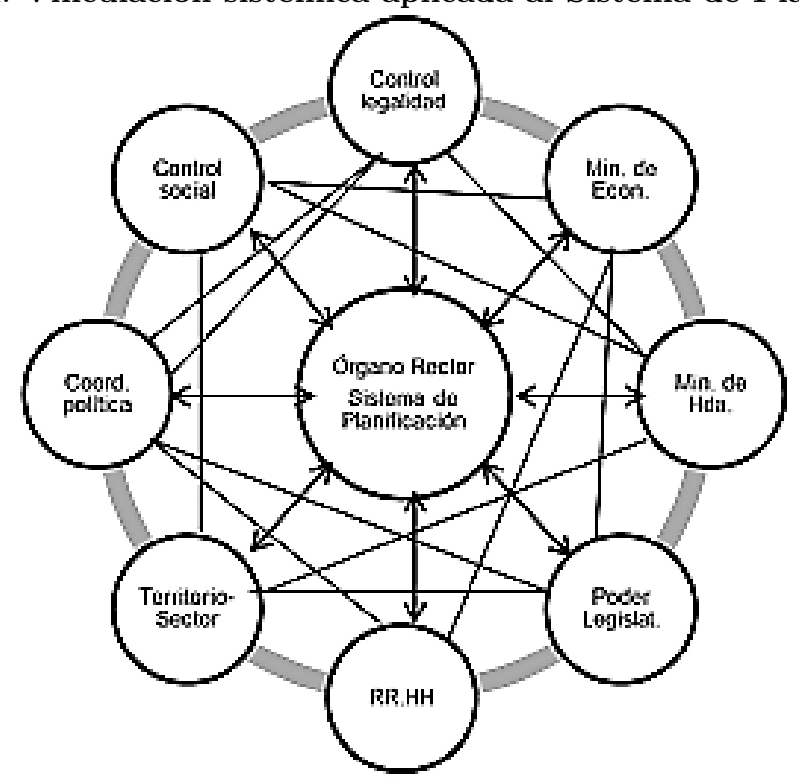

Fuente: Pacheco, 2012 
una lógica de verticalidad o de una lógica de diagonalidad.

Una lógica de verticalidad se da cuando existe un relación de subordinación entre la organización de nivel nacional con una de carácter local. En esta lógica, la estructura con alcance territorial más acotado responde a los lineamientos políticos emanados desde el organismo central, no sólo en cuanto a su funcionamiento, sino que desde el punto de vista político. Esto corresponde a una relación administrativamente desconcentrada.

Por otro lado, bajo una lógica de diagonalidad no existe una relación de dependencia y jerarquía entre el nivel central y el nivel local, ya que el último es autónomo en su actuar. La independencia del organismo local en términos políticos, no implica que haya una incomunicación entre las dos instancias en lo administrativo, sino que hay un co-desarrollo de funciones, dejando espacios de decisiones a la organización local, en la definición de sus propios lineamientos políticos y de acción. Esto corresponde a una relación administrativamente descentralizada.

Las lógicas de la verticalidad y la diagonalidad en la vinculación territorial pueden ser aplicadas de forma independiente en la relación organizacional cuando hay más de dos niveles territoriales. Es decir, el nivel central puede tener una lógica determinada de vinculación territorial con un nivel intermedio, mientras que con el nivel local puede tener una lógica distinta.

La participación ciudadana ha sido conceptualizada de diferentes maneras y con distintos grados de alcance. La relación ideal entre administración y ciudadanía, entiende la vinculación como un conjunto de actividades voluntarias mediante las cuales los miembros de una sociedad participan en la selección de sus gobernantes y, directa o indirectamente, en la elaboración, ejecución, seguimiento y evaluación de la política gubernamental (Sartori, 1989). Cabe destacar la definición entregada por Rafael González Ballar, que concibe a la participación ciudadana como "un proceso gradual mediante el cual se integra al ciudadano en forma individual o participando en forma colectiva, en la toma de decisiones, la fiscalización, control y ejecución de las acciones en los asuntos públicos y privados, que lo afectan en lo político, económico, social y ambiental para permitirle su pleno desarrollo como ser humano y el de la comunidad en que se desenvuelve" (González, 2008: 5).

Tal como lo expresa Lahera respecto de la calidad de las políticas públicas, la participación es un proceso que no sólo se circunscribe a una determinada fase de una política, sino que es transversal a todas las instancias, por tanto "una medida de participación, no hace una política de participación. Ésta se refiere a la posibilidad que personas o grupos tienen para influir, hacerse presentes en la agenda pública y también en la formulación, ejecución y evaluación de las políticas públicas" (Lahera, 2008: 106).

\section{Metodología}

La investigación se desarrolló a partir de un enfoque cualitativo de tipo exploratorio-descriptivo (Barrantes, 2002). La metodología se basó en un análisis comparativo entre el sistema de planificación de Haití, el de República Dominicana, y con un modelo teórico desarrollado por el ILPES.

Luego del análisis de la estructura del sistema de planificación, se identificaron los nodos críticos de gestión y de estructura para los niveles estratégicos, programáticos, y operativos del sistema. Para ello, se llevó a cabo una encuesta en línea a dieciocho (18) técnicos del Ministerio de Planificación de Haití, de los cuales cuatro (4) están a nivel de director. La encuesta se basó en el modelo del ILPES y fue dividida en tres secciones: a) identificación, b) órgano rector, y c) sistema de planificación de Haití.

\section{Sistema de planificación (Es- tructura típica)}

\section{Sistema de planificación}

El Sistema de Planificación es un conjunto articulado e integrado de subsistemas, órganos, principios, normas y procedimientos, que a través de la formulación y coordinación del proceso de planificación estratégica a nivel nacional, así como de la coordinación de los programas e instituciones de planificación a nivel local, tienen como objetivo promover y orientar el desarrollo económico y social del país. Un esquema general de las estructuras que conforman estos sistemas parten de una concepción de un organismo rector que tiene funciones que permiten coordinar la acción de diferentes entidades públicas relacionadas con la planificación y en particular con los organismos que implementan políticas públicas (Pacheco, 2012). 


\section{Órgano Rector}

Es el órgano central encargado de administrar el Sistema de Planificación. Su principal función es la formulación del producto del sistema de planificación, como es el caso del Plan Nacional de Desarrollo o Estrategia de Desarrollo, concebido como un instrumento de planificación que tiene por objetivo orientar un adecuado proceso de toma de decisiones, una racional asignación de los recursos y una ordenada gestión gubernamental, teniendo como referencia un horizonte temporal de mediano o largo plazo.

Otras funciones establecidas para este órgano, suelen ser la coordinación entre los planes, programas y proyectos, tanto a nivel territorial, como a nivel sectorial e institucional, así como velar por la compatibilidad de las inversiones públicas con los lineamientos generales contenidos en el Plan Nacional de Desarrollo.

En cuanto a su dirección, la tarea de órgano rector suele recaer en el Ministerio encargado de la función de Planificación, como el Ministerio de Planificación y de la Cooperación Externa en el caso de Haití.

Se manifiesta a través de un organismo encargado de coordinar los asuntos generales de Gobierno, con funciones de asesoría en los aspectos relacionados con el desarrollo económico y social del país. Este organismo define los aspectos de política pública que orientan a los organismos encargados de la dirección económica y social.

Generalmente está compuesto por el Presidente de la República, el Vicepresidente de la República y los Ministros de Estado, en calidad de miembros permanentes, y también por otros miembros no permanentes dependiendo de la naturaleza de la discusión y las temáticas a tratar. A nivel jerárquico, se sitúan fuera de la dependencia de las carteras particulares de Planificación y suelen vincularse de manera directa con la Presidencia bajo la forma de organismo asesor.

Son los entes encargados de la planificación a nivel territorial o a nivel sectorial, a través de Unidades o departamentos de Planificación en los distintos organismos públicos que contribuyen al desarrollo del sector social determinado o al área geográfica de aplicación. Estas unidades se encuentran en los Ministerios, en las instituciones descentralizadas y en las entidades públicas regionales y locales ?Unidades de Planificación Institucional (UPI‘s) ?. Esta instancia se puede delegar a determinados agentes económicos y sociales a nivel territorial, los cuales tienen como función canalizar las demandas ciuda- danas hacia al nivel administrativo que corresponda, participando en la formulación de los planes estratégicos territoriales, según la unidad administrativa pertinente.

El eje de apoyo técnico se plasma con mayor claridad en otras instancias, a través de la vinculación de las funciones de planificación con los sistemas interrelacionados, como es el caso de los Sistemas de Presupuesto, de Crédito Público, de Tesorería, de Contabilidad Gubernamental y de Control.

Al comprender a la planificación como una actividad de interrelación, cabe destacar cómo ciertos sistemas de planificación contemplan instancias específicas que permiten vincular y formar alianzas interinstitucionales, público-privadas o de otra índole, de manera de mejorar la coordinación y asesoría en aspectos particulares.

Este esquema presenta en forma general la composición de los Sistemas Nacionales de Planificación. Es posible generalizar la presencia de determinados ejes comunes a los distintos sistemas de planificación analizados, lo que hace posible enmarcar al Sistema de Planificación bajo la teoría general de sistemas en organizaciones propuesta por Katz y Khan, adecuando la función de los ejes descritos a la interrelación con otros sistemas.

\section{Ámbitos}

Con el fin de identificar las tareas que realiza tanto el órgano rector como los distintos organismos públicos relacionados/vinculados dentro del sistema, se describe lo que llamaremos ámbitos de acción o ámbitos. Estos ámbitos nos dan una visión de las preocupaciones actuales de los sistemas de planificación.

\section{Planificación Económica y social}

El área de política económica y social dice relación con la coordinación de los planes y programas de los distintos sectores de intervención gubernamentales con el Plan Nacional de Desarrollo. Los sectores son familias de problemas económicos y/o sociales interrelacionados entre sí, por medio de una especialidad del conocimiento técnico-científico que conforman un criterio convencional de organización del gobierno. Así, los sectores tienen su expresión en Ministerios sectoriales específicos.

Para asegurar la coherencia de la planificación sectorial con el sistema nacional de planificación, se establecen metodologías técnicas y lineamientos políticos y normativos a partir del plan nacional 
de desarrollo, que emanan del órgano rector a los ministerios. Además se requiere la realización de estudios que permitan establecer e incluir dentro del proceso de planificación las particularidades del sector, previendo sus futuros cambios. Es por ello que las principales temáticas del área de política económica y social son 1) Coordinación Institucional; 2) Estudios Prospectivos; y 3) Planificación y Metodología.

Esta área debe ubicarse en una unidad diferenciada dentro del núcleo operativo del órgano rector, contando con dispositivos de enlace que lo conecten directamente con el proceso de planificación sectorial. Este dispositivo son las unidades de planificación insertas en la estructura organizativa de los ministerios sectoriales que ésta unidad diferenciada deberá coordinar.

\section{Desarrollo Territorial}

El área de desarrollo territorial (nivel Intermedio o Local) comprende la coordinación y elaboración de criterios técnicos y políticos para la elaboración e implementación de planes de desarrollo territoriales y el ordenamiento del territorio procurando la complementariedad entre niveles. Esta área comprende dos temáticas diferenciadas, la coordinación de la planificación territorial y el ordenamiento territorial.

La coordinación de la planificación territorial se refiere a la gestión del proceso de planificación en cada unidad territorial que sea pertinente. Comprende: 1) Asistencia técnica y metodológica para la elaboración de planes territoriales; 2) Definir lineamientos políticos, conceptuales y normativos que permitan focalizar las políticas territoriales; 3) Generar información sobre las políticas territoriales vinculadas al proceso de planificación; y 4) Coordinar los planes y programas de inversión entre niveles territoriales.

El ordenamiento territorial dice relación con la territorialización de las políticas locales, estableciendo clasificaciones zonales, y en base a estas, priorizaciones para la planificación e inversión pública, objetivos específicos de política local, regulaciones espaciales de uso de recursos y prácticas culturales, etc. El proceso de ordenamiento territorial debe estar integrado al proceso de planificación territorial, por lo que ambas temáticas están incluidas en una misma área, que por lo general está a cargo de una unidad diferenciada dentro del órgano rector del Sistema Nacional de Inversión.

\section{Inversión Pública}

El área de inversión pública comprende la rectoría del sistema nacional de proyectos públicos de inversión derivados de los planes de desarrollo, desde la formulación de los proyectos hasta su evaluación. El proceso de inversión pública debe contar al menos con las siguientes etapas: 1) Preinversión; 2) Priorización; 3) Programación; 4) Ejecución; y 5) Evaluación. Esas cinco etapas, si bien se gestionan de forma descentralizada dependiendo de la unidad territorial y sector, son coordinadas y dirigidas desde una unidad especializada del órgano rector.

Con el fin de maximizar la eficiencia y eficacia en la gestión del área es necesario dar seguimiento al cumplimiento de los proyectos estableciendo un sistema de información de la inversión pública requiriendo coordinación con las unidades sectoriales y territoriales y también gestionando desde la misma unidad especializada.

Los sistemas de inversión permiten darle consistencia al proceso de diseño de planes a través de la vinculación de éstos a los distintos presupuestos del Estado, por lo tanto la vinculación entre presupuesto y plan es fundamental.

\section{Modernización del Estado}

El ámbito de Modernización del Estado rige aquellos procesos a través de los que la organización del aparato burocrático del Estado es modificada de manera deliberada para aumentar sus grados de eficiencia y eficacia, adaptándola a las necesidades impuestas por los objetivos políticos y de gestión derivados del Sistema Nacional de Planificación. Este ámbito abarca dos temáticas, Rediseño Institucional y Fortalecimiento Institucional.

La temática de Rediseño Institucional dice relación con los cambios en las estructuras organizativas del gobierno y en las relaciones entre unidades, tales como descentralización, desconcentración de servicios y la creación, eliminación, rediseño y fusión de unidades. El fortalecimiento institucional en cambio, dice relación con aquellas políticas destinadas a mejorar la gestión del Estado, a través de la introducción de cambios destinados a optimizar la eficiencia administrativa dentro de la gestión estratégica del Estado, en áreas tales como recursos humanos, finanzas, contrataciones públicas, política de incentivos, abastecimientos, etc.

Debe existir una unidad dentro del órgano rector del Sistema Nacional de Planificación encargado de dirigir los procesos de "Reforma" y "Fortalecimiento" institucional del Estado. Esta unidad debiese 
encargarse de abordar las tres temáticas del área: 1) Fortalecimiento Institucional; 2) Rediseño Institucional; y 3) Estudios de diagnóstico de la gestión del Estado.

\section{Cooperación Internacional}

El ámbito de cooperación internacional puede definirse como aquel ámbito encargado de coordinar y canalizar la oferta de cooperación en programas y proyectos de inversión; así como de asistencia técnica, compatibilizándola con las necesidades de cooperación derivadas de los planes de desarrollo vigentes y atrayéndola hacia las áreas técnica y políticamente prioritarias.

Este ámbito abarca la captación, aprobación, programación, seguimiento y evaluación de todos los programas y proyectos de inversión provenientes de fuentes internacionales, sean de carácter bilateral (provenientes de países específicos) como multilateral (provenientes de organizaciones internacionales). Este ámbito también integra la cooperación de carácter técnico-metodológico y las asesorías que distintos gobiernos, ONGs y organismos internacionales ofrecen a los gobiernos nacionales. Para ello, todos estos elementos deben ser incorporados en una sola unidad dentro de la estructura del órgano rector del sistema nacional de planificación.

En este ámbito es donde el Plan Nacional de Desarrollo debe converger con la política internacional de los gobiernos, por tanto se requiere de una coordinación directa con el ministerio encargado de las relaciones exteriores e internacionales del país. La coordinación de la política de cooperación internacional en el contexto de la planificación del desarrollo, requiere la concurrencia del ministerio de relaciones exteriores/internacionales, el órgano rector del sistema nacional de planificación, el ministerio de economía/finanzas y presidencia, a través de una instancia a modo de "consejo de cooperación" que pueda articular las políticas sectoriales con las necesidades y objetivos de la planificación.

\section{Evaluación}

El ámbito de evaluación puede definirse como aquel que rige las actividades dentro del sistema nacional de planificación, encargadas de determinar el grado de eficiencia, eficacia y efectividad en la ejecución de planes, programas y proyectos gubernamentales vinculados con los planes de desarrollos vigentes en cada país. Éste ámbito abarca dos temáticas distintas, evaluación y seguimiento.

La evaluación implica la aplicación de metodo- logías específicas de evaluación para cada caso, de tal manera de establecer cumplimientos, desviaciones y aprendizajes. El monitoreo establece el grado de avance de la implementación y/o ejecución de un programa o proyecto público. El monitoreo es una actividad de coordinación, que se realiza de forma conjunta e implica la generación de un sistema de información que vincule a la unidad encargada del monitoreo con las distintas unidades de ejecución.

Sin embargo, las tareas de evaluación y seguimiento deben quedar claramente definidas en una sola unidad diferenciada dentro del órgano rector. Es necesaria la coordinación con las oficinas de estadística e información pública, ya que el principal insumo de las unidades de evaluación y seguimiento será el emanado de estas; la coordinación con la unidad de inversión pública, para no incurrir en duplicación de funciones; y además es necesario integrar el sistema de información sobre proyectos de inversión a un sistema general de información pública. Finalmente, es necesaria la coordinación con la unidad encargada de la modernización del Estado, de tal manera de que no exista una duplicación de tareas en la evaluación de la eficiencia, y eficacia del aparato público. El ámbito de evaluación se relaciona directamente con los objetivos que emanan de los distintos planes de desarrollo vigentes.

\section{Capacitación}

El ámbito de capacitación abarca todos aquellos procesos que buscan mejorar la calificación de los recursos humanos existentes vinculados con el Sistema Nacional de Planificación, de tal manera de dotarlos de los conocimientos técnicos, legales y políticos necesarios para lograr una mejora en el desempeño de sus funciones dentro del mismo.

La capacitación debe responder a las necesidades del sistema de planificación. Debe constituirse como una unidad diferenciada dentro del núcleo operativo del órgano rector del Sistema Nacional de Planificación, tendiendo un rol de apoyo para los demás ámbitos, proveyendo de programas de capacitación de acuerdo con sus necesidades y metodologías de trabajo. Por lo tanto, se debe coordinar con todas las demás unidades dentro del órgano rector.

\section{Instrumentos}

El Sistema Nacional de Planificación requiere, para su funcionamiento coordinado y eficiente, de una serie de instrumentos que introduzcan mayor racionalidad a la acción previsora de los gobiernos y sus distintos niveles institucionales. Se debe agre- 
gar la visión estratégica inserta en la planificación, la cual contempla una lógica direccionada para conseguir determinados resultados que logren la situación ideal a alcanzar.

Bajo este esfuerzo racionalizador y estratégico, a continuación, se propone el uso de instrumentos para cada nivel de gobierno, expresados en planes, presupuestos, instrumentos procedimentales para el funcionamiento de los planes e instrumentos tecnológicos de gestión de información de los mismos, organizados y compuestos de la siguiente forma:

- Visión de Desarrollo País: Consiste en la imagen objetivo de un país, hacia donde se quiere ir o el estado de desarrollo a alcanzar por una nación, pensada para el largo plazo. Esta visión se expresa en un programa de gobierno, pero debe ser debatida y consultada por los diversos actores sociales y políticos para lograr un consenso respecto de las orientaciones estratégicas que se definirán en un país para alcanzar dicha visón.

- Plan de Desarrollo Nacional: Principal instrumento orientador de la planificación de un país con visión prospectiva. Contiene las prioridades estratégicas respecto de los objetivos de mediano y largo plazo en materias políticas, económicas, de desarrollo y medioambientales, dentro de los cuales se deberán enmarcar el resto de los instrumentos y mecanismos de planificación.

- Planes sectoriales: Planes formulados respecto a un determinado sector o área de políticas como educación, salud, trabajo, etc., coordinados y sistematizados por el respectivo ministerio o secretaría de estado.

- Planes de desarrollo territorial: Instrumentos para definir el accionar de los gobiernos subnacionales respecto del desarrollo regional y local y la programación de sus respectivas inversiones. Estos planes se relacionan tanto con la planificación nacional como con la sectorial, en un esfuerzo de coordinación que contribuya a cumplir las metas nacionales fijadas.

- Planes de desarrollo institucionales: Son los planes elaborados por las instituciones, organismos y servicios del gobierno nacional para la definición de sus presupuestos, planes estratégicos y planes de acción de carácter anual en sus materias tomando en consideración la visión, misión, objetivos y metas que se establecen para cada institución.

- Presupuestos nacionales y territoriales: Instrumentos para captar y asignar recursos, bajo un proceso de formulación, discusión y aprobación, ejecución, control y evaluación. Es la expresión financiera de los planes nacionales y territoriales, programas, políticas y proyectos. Sientan las bases para que cada ente administrativo pueda llevar a cabo las funciones que les son encomendadas por ley, bajo un proceso de gestión eficiente y transparente. Dependiendo de la naturaleza de la planificación y de las acciones que debe realizar cada organismo, los presupuestos pueden tener plazos anuales o plurianuales.

- Instrumentos procedimentales: Son todas aquellas herramientas para regular, poner en marcha y procesar los planes en sus distintos niveles. Este es el caso de leyes y otras normativas de planificación, metodologías para formular planes y proyectos de inversión, manuales y guías para la construcción de estrategias institucionales o de desarrollo territorial.

- Instrumentos tecnológicos para la información y transparencia: Son los instrumentos para apoyar la gestión de los planes, con los respaldos de información para la evaluación y monitoreo tanto interna como externa y para la ciudadanía, de carácter sistémico e integrado en una lógica de gobierno electrónico como sistemas informáticos de gestión financiera y presupuestaria de los planes, cuadros indicadores, sistemas de información y atención ciudadana, sistemas nacionales de compras y adquisiciones públicas y portales electrónicos de licitaciones públicas.

\section{Mecanismos de articulación - coordi- nación del SNP}

Los mecanismos de coordinación son aquellos que integran en organismos colegiados a representantes de distintas organizaciones o de unidades administrativas provenientes de los sectores políticos, empresariales y de la sociedad civil, para lograr alinear su accionar a los lineamientos de la planificación nacional.

\section{Los mecanismos de articulación / coordina- ción políticos}

Estos se expresan en las instancias colegiadas como consejos de carácter presidencial o ministerial para organizar los grandes ejes temáticos o sectoriales en los cuales se estructura la planificación, estipulando sus vínculos con el poder legislativo cuando esto corresponda, definiendo las respectivas responsabilidades en relación con la aprobación 
de los planes nacionales. En el caso territorial se sigue una lógica similar, adecuando las responsabilidades a las autoridades locales, constituyendo para su efecto consejos de gobierno territoriales e instancias de discusión entre las máximas autoridades regionales, departamentales o estaduales con alcaldes y regidores.

\section{Mecanismos articulación / coordinación técnicos}

La coordinación técnica depende de organismos colegiados de consulta y revisión, compuestos por integrantes del área técnica del gobierno. Su función consiste en analizar los planes, contrastando los lineamientos y acciones a convenir con variables de crecimiento económico, panoramas de la economía mundial y situación socio-económico actual del país.

\section{Los mecanismos articulación / coordinación} sociales

Estos mecanismos están relacionados con aquellas instancias de consulta y participación ciudadana, relacionadas con la definición de planes y estrategias ya sea a nivel local y regional o en representación de áreas de interés transversal para el país. Es posible definir mecanismos de consulta directa a la comunidad para establecer las prioridades del plan a nivel territorial, o institucionalizar instancias colegiadas para la discusión de los planes nacionales a través de la interacción directa con las autoridades gubernamentales.

\section{Dispositivos de Enlace del SNP}

Estas son estructuras o personas que pertenecen a un organismo en particular y que tienen la responsabilidad o función de vincularse como contraparte de otro organismo dentro del sistema de planificación.

\section{Sistema de planificación de la República Dominicana}

\section{La estructura del órgano rector de la República Dominicana}

En diciembre del año 2006 el Congreso Nacional dominicano aprobó la Ley del Sistema Nacional de Planificación e Inversión Pública y la Ley que crea la Secretaría de Estado de Economía, Planificación y Desarrollo (SEEPYD), con el objetivo de continuar el proceso de modernización del Estado. La SEEPYD es el Órgano Rector del Sistema Nacional de Planificación e Inversión Pública. Tiene la misión de conducir y coordinar el proceso de formulación, gestión, seguimiento y evaluación de las políticas macroeconómicas y de desarrollo sostenible para la obtención de la cohesión económica, social, territorial e institucional de la nación. Está conformada por la Subsecretaría de Estado de Planificación, la Subsecretaría de Estado de Cooperación Internacional y la Subsecretaría de Estado Técnico Administrativa y está diseñado según el siguiente esquema.

\section{El sistema Nacional de la Planificación y de la Inversión Pública}

El Sistema Nacional de Planificación e Inversión Pública está integrado por el conjunto de principios, normas, órganos y procesos a través de los cuales se fijan las políticas, objetivos, metas y prioridades del desarrollo económico y social evaluando su cumplimiento. Está relacionado con los Sistemas de Presupuesto, Crédito Público, Tesorería, Contabilidad Gubernamental, Compras y Contrataciones, Administración de Recursos Humanos, Administración de Bienes Nacionales y Control Interno.

El Sistema de Planificación e Inversión Pública está integrado por: Consejo de Gobierno, Comisión Técnica Delegada, Secretaría de Estado de Planificación y Desarrollo, Consejo de Desarrollo Regional, Consejo de Desarrollo Provincial, Consejo de Desarrollo Municipal.

\section{Sistema nacional de planifica- ción de Haití}

\section{La Estructura del Órgano Rector de Haití}

La misión del Ministerio de Planificación de Haití es orientar, fomentar y apoyar el desarrollo nacional y local en su dimensión económica, social, cultural y territorial y para aumentar la eficacia de las iniciativas que le conciernen. En sus intervenciones, toma en cuenta las características específicas del país y promueve el crecimiento económico, la creación de empleo y la lucha contra la pobreza, buscando la igualdad entre mujeres y hombres.

La estructura del órgano rector cuenta con seis 
Direcciones Técnicas y cuatro Unidades dependiente de la Dirección General que son: Dirección de la Planificación Económica y Social, Dirección del Ordenamiento del Territorio, del Desarrollo Local y Regional, Dirección de la Cooperación Externa, Dirección de la Inversión Pública, Dirección de Monitoreo y de Evaluación, Dirección de los Asuntos Administrativos, del Presupuesto y de los Recursos Humanos, Unidad de Estudios y de Programación, Unidad de Coordinación de las Direcciones Departamentales, Unidad de Comunicación, de Informática y de la Documentación u Unidad de Coordinación de las Actividades de los ONG.

\section{El Sistema de Planificación de Haití}

Ante la ausencia de una ley de planificación que plantee de manera global todos los actores interviniendo en el sistema de planificación haitiano, se ha formalizado la estructura de la planificación de Haití basada en el modelo de planificación que se estaba implementando en los años previos al terremoto del 12 de enero de 2010, al marco legal de la constitución de la República de Haití y por ende al modelo de estructura según el enfoque sistémico desarrollado por el ILPES. De esta manera la estructura de planificación quedó configurada de la siguiente manera:

- Un Órgano Rector que elabora planes nacionales de desarrollo económico y social; y mejora los sistemas de planificación, permitiendo la utilización óptima de los recursos disponibles para un desarrollo equilibrado económico y social.

- Una estructura de adaptación, compuesta por la Presidencia y el Primer Ministro, que concibe, define, elabora e implementa las grandes opciones de política gubernamental.

- Una estructura de mantenimiento, conformado por el Poder Legislativo; la Contraloría de la República; y el Ministerio de Economía y Finanzas, que asegura de las normas de subsistencia del sistema.

- Una estructura de apoyo, formada por la cooperación internacional, que juega un papel muy importante en el financiamiento de los proyectos de desarrollo y las distintas organizaciones de la sociedad civil que contribuyen a la tarea de planificación del desarrollo en Haití.

- Una estructura de producción en la cual los distintos ministerios sectoriales entregan productos y servicios a la ciudadanía. Existen Comités Interministeriales, que permiten a los distintos ministerios llevar a cabo políticas públicas en uno o varios campos conexos de manera coordinada y armónica.

- Un sistema de vinculación territorial, el cual, junto con el Ejecutivo, estudia y planea los proyectos de descentralización y desarrollo del país, desde el punto de vista social, económico, comercial, agrícola e industrial. Con el fin de favorecer la participación y el desarrollo regional y local se crearon algunos mecanismos de participación ciudadana bajo el liderazgo del gerente de dichas instituciones, denominados Consejo de desarrollo del Departamento, de la Comuna o de la Sección Comunal.

El Sistema nacional de planificación y de gestión del desarrollo se dirigía a la implementación de un marco administrativo y asociativo para la planificación, la programación, el financiamiento, la implementación, la valuación y el control de las actividades de desarrollo y de ordenamiento del territorio.

Varias iniciativas gubernamentales basadas en los fundamentos de la gobernanza y del pilotaje estratégico estaban en proceso de definición y/o de realización. De hecho, el desarrollo y la implementación gradual del sistema nacional de planificación y de gestión del desarrollo integraba la definición y la puesta en ejecución gradual de varias medidas interrelacionadas y agrupadas en cinco subsistemas del sistema nacional de planificación y de gestión del desarrollo. Estos subsistemas son: Subsistema de Planificación del Desarrollo y del Ordenamiento del Territorio (SSPDOT), Subsistema de Financiación del Desarrollo y del Ordenamiento del Territorio (SSFDOT), Subsistema de Gestión de la Inversión Pública (SSGIP), Subsistema de Asociación Estado-sociedad civil en el Desarrollo y el Ordenamiento del Territorio (SSADOT) y Subsistema de Informaciones sobre la Planificación y la Gestión del Desarrollo (SSIPDG).

El SSPDOT debía permitir la orientación, la estructuración y la estimulación de los esfuerzos nacionales y locales de desarrollo y de ordenamiento del territorio, del mismo modo administrar la utilización del territorio, de los recursos naturales y determinar las herramientas y los mecanismos necesarios a la planificación, a la gestión del desarrollo y al ordenamiento del territorio.

El SSFDOT debía permitir movilizar y aumentar los recursos disponibles tanto internos como externos, canalizándolos sobre las orientaciones y los objetivos perseguidos, asimismo repartir las posibilidades de desarrollo sobre el conjunto del territorio, valorizar los grandes potenciales y del mismo modo levantar las grandes limitaciones al desarrollo de 
Haití, y de la misma manera, sostener la acción de los socios nacionales y locales del desarrollo.

El SSGIP debía permitir a aumentar la pertinencia y la transparencia de las inversiones públicas, igualmente guiar cada año la elaboración, la implementación, la evaluación y el seguimiento de las repercusiones del Programa de Inversión Pública (PIP) y adicionalmente responsabilizar a todos los actores públicos del desarrollo y del ordenamiento del territorio en cuanto a sus respectivos ámbitos.

El SSADOT debía permitir la movilización coordinada de los actores para el crecimiento económico, la creación de empleo, la lucha contra la pobreza, y el mejoramiento de la asistencia en los servicios públicos así como la utilización racional, la gestión sostenible del territorio y los recursos naturales.

El SSIPDG debía permitir la caracterización, la actualización periódica del estado de desarrollo, el mejoramiento de las metas de las inversiones públicas y privadas, la gestión de los programas y de los proyectos en curso de realización o programados, la visualización de las desviaciones entre el estado de desarrollo y las orientaciones y los objetivos perseguidos así como la difusión de estas informaciones.

\section{Descripción y análisis de los re- sultados}

\section{Descripción de la tabla de compara- ción de los sistemas de planificación}

Para diseñar esta tabla de comparación, se tuvo como referencia el modelo del sistema de planificación del ILPES. Al respecto, hemos planteado la estructura de la tabla en dos ejes de lo cual se destaca una parte para el órgano rector y la otra parte para el sistema de planificación. A cada una de ella se le asocian cinco (5) columnas respectivamente para las variables, la estructura de planificación de Haití, la de la República Dominicana, la de la estructura típica, la otra para la calificación. Adicionalmente, en las filas se plantean los componentes esenciales del sistema de planificación del modelo teórico. La idea es comparar la estructura de la República de Haití y la estructura de la República Dominica con este modelo teórico del ILPES. Si la estructura funciona y existe en el marco legal le atribuimos la nota 7 ; asimismo, si no funciona en la realidad y existe en el marco legal por tanto concluimos la existencia de la estructura con una nota 7 pero cuando no existe en la práctica ni en el marco legal lo calificamos con la nota 1 lo que se refiere a una experiencia que no se realiza. Así, la calificación para la existencia se basa en dos notas 1 y 7 .

\section{Análisis de los resultados de la tabla de comparación}

Considerando las Direcciones del Ministerio de Planificación de Haití, falta una Dirección de Desarrollo Institucional que podría ser muy importante para esa estructura, en la medida que pueda formular, implementar y controlar proyectos que busquen el desarrollo de largo plazo de la institución, con el fin de responder de manera más eficiente y mejor a las necesidades actuales y futuras de la organización.

El Ministerio cuenta con una Dirección de Inversión Pública que se encarga del Programa de Inversión Pública, el cual es un instrumento muy importante en todo el ciclo del proceso de los proyectos de desarrollo. Igualmente, cuenta con un Dirección de Planificación Territorial y una Dirección de Monitoreo y Evaluación que hace el seguimiento y la evaluación de los proyectos de desarrollo para que estos alcancen a sus metas.

Con respecto a las funciones de un sistema de planificación, de acuerdo a la ley orgánica y a las distintas acciones emprendidas por el Ministerio, se puede aseverar que este último está cumpliendo sus distintas funciones, tanto de formulación de la estrategia y del plan de desarrollo como la de la coordinación de los planes, programas e institucional. Es necesario precisar que todas las funciones no están siendo ejercidas con el mismo grado de avance o de eficacia.

Asimismo, se puede apreciar que el ministerio mantiene relación con los distintos ejes de coordinación, según lo establecido en la ley y lo observado en la práctica a través de las distintas reuniones organizadas con este fin. Con respecto a lo anterior, el ministerio no se relaciona con los distintos ejes de coordinación de la misma manera. Hay relaciones que son mucho más estrechas que otras.

Globalmente, con respecto a los componentes de la teoría general de sistemas en organizaciones, se destacaron los cincos grandes ejes de la teoría general de sistema en organizaciones, por lo cual el Ministerio de Planificación de Haití, actuando como una estructura gerencial, se interrelaciona con una estructura de adaptación, de producción, de mantenimiento y de apoyo.

Dentro de los ámbitos de acciones, al sistema nacional de planificación de Haití le falta una Dirección de Modernización del Estado, lo que puede ser 
un impedimento mayor, particularmente al objetivo de adaptar cambios necesarios en el aparato público tendiente a aumentar el grado de eficacia y eficiencia en el alcance de sus objetivos. Cabe mencionar que esta estructura existe en la Primature ${ }^{1}$, pero pensamos que sería mejor integrarlo al sistema de planificación, lo que podría darle una orientación mucho más técnica, alineándola con un enfoque de desarrollo. Los otros ámbitos de acciones existen y funcionan.

El sistema de planificación de Haití cuenta con todos los instrumentos propuestos por el modelo teórico, exceptuando el plan de desarrollo institucional, con el cual el ministerio podría adecuar la evolución de las condiciones externas con las condiciones internas, asegurando la vigencia y la pertinencia de la institución con respecto a su orientación estratégica. El sistema tampoco cuenta con un presupuesto territorial, lo que es una herramienta muy importante para la descentralización. Esto puede generar ineficiencias en la distribución y la coordinación de los flujos de financiamiento de los proyectos regionales, concentrando los recursos en algunas regiones, particularmente en la capital, y produciendo un desarrollo desequilibrado.

Al mismo tiempo, no existe una estructura de comisiones de política fiscal, la que podría permitir una armonización del Plan Nacional de Desarrollo con la estructura de la programación presupuestaria. Eso podría explicar, en parte, que hoy en día la mayoría de los proyectos de desarrollo estén financiados con ayuda externa. Por lo tanto, no se han desarrollo mecanismos a través de los cuales la política fiscal puede jugar un rol vinculante entre los Planes de Desarrollo y la base imponible, es decir que no existen mecanismos de incentivos para generar mayor ingreso. Tampoco existen dispositivos de enlace institucional, lo que significa que no hay un vínculo estrecho entre el plan nacional y los planes sectoriales y territoriales, lo cual puede generar un problema de coordinación en la implementación de los proyectos.

\section{Descripción de la encuesta}

La encuesta tiene tres (3) secciones. La primera corresponde a la identificación, la segunda a las preguntas relativas a la estructura del ministerio y la tercera al sistema de planificación. Esta última consiste en veintidós (22) preguntas cerradas de las cuales los encuestados tienen que evaluar los distintos elementos del sistema según un escala, donde a

\footnotetext{
${ }^{1}$ La Primature es el órgano gubernamental dirigido por el Primer Ministro asegurando funciones políticas, administrativas y técnicas.
}

la nota uno (1) le corresponde a una experiencia que no exista o no se realice, la nota tres (3) se asignará cuando existe el marco legal no implementado, la cinco (5) cuando la experiencia se realiza de acuerdo con los estándares definidos y la siete (7) cuando se refiere a una experiencia exitosa.

\section{Análisis de los Resultados de la En- cuesta}

Se destaca una nota promedio obtenida de 4 para las direcciones de Planificación Económica y Social, la Dirección de la Inversión Pública, la Dirección de Monitoreo y Evaluación y la Dirección de los Asuntos Administrativos; lo que significa que, en la percepción de los encuestados, estas Direcciones funcionan bastante bien, pero no de acuerdo con los estándares definidos. Sin embargo, la Dirección de la Cooperación Externa y la Dirección del Ordenamiento del Territorio no son bien percibidas. La mala percepción de la cooperación internacional tiene que ver con la fuerte dependencia que existe entre la cooperación internacional y el Estado Haitiano en cuanto al capricho del financiamiento para el desarrollo y además al fracaso de la cooperación al desarrollo que no ha podido arrancar conjunto con el Estado haitiano un verdadero proceso de desarrollo después de los últimos dos décadas. En cuanto a la dirección del ordenamiento del territorio, tiene relación con el proceso anárquico de centralización de las ac ividades económicas en la capital y el débil desarrollo de las actividades en las regiones. Existe un problema de gestión en este nivel, por lo que muchos esfuerzos deben ser desarrollados en esta instancia para que el ordenamiento del territorio y la descentralización puedan jugar un rol mucho mayor en la distribución del ingreso.

Un porcentaje de $83 \%$ de los empleados del ministerio estiman que sería necesaria Dirección de Desarrollo Institucional en la estructura del Ministerio de Planificación de Haití, con el fin de desarrollar programas y proyectos para el sistema de planificación.

Por su parte, comparativamente con las direcciones, las unidades no fueron muy bien percibidas, dado que solamente la Unidad de Coordinación de las Actividades de las ONGs consigue una nota de 4.44. Esta percepción puede ser justa en la medida en que se considere la proliferación de ONGs en las últimas dos décadas; pero este nivel de satisfacción es relativa cuando se observa que un gran parte de las ONGs que desarrollan proyectos de desarrollo, siguen funcionando fuera del control de la unidad. En contraposición, la Unidad de Estudios y Pro- 
gramaciones (UEP) es mal percibida, lo que constituye un impedimento enorme para los estudios, análisis e investigación que requiere la eficacidad y la eficiencia del funcionamiento de todo sistema de planificación.

Dentro de las funciones que desarrolla el Ministerio de Planificación, la de formulación de estrategia y la de formulación del plan son las que se cumplen mejor. Eso constituye un buen punto, en la medida en que eso permitiría orientar racionalmente las actividades de desarrollo a largo plazo. En cambio, la coordinación institucional se realiza con menor eficiencia, lo que podría resultar en un conflicto de roles entre las instituciones que actúan dentro del mismo ámbito, como es el caso del Ministerio del Medio Ambiente y el Ministerio de Agricultura, que a veces no coordinan sus acciones e intervienen sobre un mismo asunto.

El Ministerio de Planificación cuenta con una relación mucho más estrecha con el sector político en cuanto a los estándares típicos establecidos, lo cual puede tener un alto impacto, tanto positivo como negativo, en el funcionamiento del ministerio. Es decir que a través de este vínculo los partidos políticos pueden canalizar y concretizar mejor su proyecto de sociedad por intermedio del ministerio, o bien aprovechar la cartera de proyectos del ministerio para politizar.

En segundo lugar viene la relación que el ministerio mantiene con el apoyo técnico, lo que resulta claro cuando se sabe que el Ministerio de Planificación junto con el Ministerio de Economía y Finanzas (Ministerio de Hacienda) realizan distintas actividades, como la de elaborar el proyecto de ley del presupuesto nacional, el cual es un instrumento muy importante para el financiamiento de los Programas de Inversión Pública (PIP). Sin embargo, la relación que mantiene el ministerio con las otras instituciones y a nivel territorial, es defectuosa considerando la percepción que tienen los empleados de esta institución. Siendo así las cosas, se puede generar una desaceleración del proceso de desarrollo considerando el rol que el ministerio debe jugar en las regiones con los distintos sectores.

La coordinación interna es mal percibida al interior del Ministerio de Planificación, lo que puede resultar de la concentración de algunas tareas en la cúspide de la organización, reflejando una estructura vertical. En cambio, la coordinación que el ministerio establece con las demás instituciones, particularmente con los ministerios sectoriales, es mejor que la coordinación interna. Esto resulta razonable por el hecho de que las demás instituciones no dependen del ministerio y están ubicadas al mismo nivel jerárquico que el Ministerio de Planificación, lo que favorece una relación de tipo horizontal que a su vez permite una coordinación mucho mejor.

La gestión que está haciendo el ministerio saca un promedio de 3.33 de los encuestados, es decir que la percepción que los encuestados tienen de la gestión de este órgano está por debajo del promedio. Esto puede significar que, a nivel estratégico, la gestión del ministerio no se extiende hacia el conjunto de trámites que el ministerio debería llevar a cabo para resolver un asunto o concretar su proyecto; o, a nivel de la cultura de la organización, la existencia de una falla en el grupo de acciones que debería promover los valores del proyecto en cuestión; o, a nivel operacional, la dificultad de realizar adecuadamente o de aterrizar las decisiones. No sabemos si esta insatisfacción viene de la estructura del ministerio, bajo el concepto de que el mal funcionamiento puede ocurrir a causa de que no hay una buena comunicación entre los distintos departamentos. Por último, no sabemos si esta percepción puede provenir de la ejecución, es decir, si eso consiste en no tomar las decisiones adecuadas y oportunas, o no fomentar la mejora de la productividad y/o no entregar el valor público a la ciudadanía.

Los ámbitos de acciones del ministerio tampoco no están funcionando bien. Dentro de estos ámbitos de acciones tres de ellos sacaron un promedio de 4: el de la cooperación internacional, el de la planificación económica y social y el de la inversión pública. Esto se relaciona con el rol que le toca al ministerio a jugar respecto de la ayuda externa que está recibiendo el país para inversiones en proyectos económicos y sociales. En cambio, la capacitación no es bien percibida por parte de los encuestados, ya que según esa misma percepción el ministerio no está desarrollando una política pública en materia de capacitación correspondiente al objetivo de la planificación y del desarrollo.

No cabe duda, de que es muy importante una Dirección de Modernización del Estado dentro del Sistema de Planificación de Haití, puesto que un porcentaje del $89 \%$ de ellos piensa que es conveniente para aumentar sus grados de eficiencia y de eficacia a toda la administración pública. En cambio, a pesar de que esta estructura existe en la Primature, un porcentaje de $72 \%$ respondió que no existe una estructura parecida en la administración pública, frente a $17 \%$ que opinan que sí y el $11 \%$ que no sabe.

Con respecto al Sistema de Planificación de Haití, solamente los instrumentos rectores, es decir, la Visión de Desarrollo del País y el Plan Nacional de Desarrollo obtuvieron un punto que esta levemente por sobre el promedio, respectivamente $4.22 \mathrm{y}$ 4.28. Esto puede constituir una dificultad mayor 
para el Sistema de Planificación en la medida en que la falta de esos instrumentos puede impactar negativamente en el esfuerzo racionalizador de las actividades del ministerio y sobre todo los medios destinados a alcanzar los objetivos fijados.

En suma, la coordinación del Ministerio de Planificación con algunos actores relevantes del sistema se encuentra en una situación precaria, considerando la percepción de los empleados de acuerdo a los datos de la encuesta, salvo la coordinación a nivel del consejo de gobierno que obtuvo una nota de 5.22. En cambio, en las otras estructuras de coordinación, tanto a nivel nacional como a nivel regional, sectorial e institucional, la evaluación fue muy mala, con notas que van desde 2 a 3 . Dentro de los componentes de la estructura del sistema de planificación, los mecanismos de articulación del Sistema de Planificación de Haití fueron los peores evaluados. Es razonable entonces afirmar que existe un problema real y que se hace necesario intervenir o incentivar la participación de los actores relevantes en el proceso de toma de decisiones, como por ejemplo los sectores políticos, empresariales y la participación ciudadana.

De acuerdo a la percepción de los encuestados del ministerio, resulta que en la práctica no existe un hábito de consulta ciudadana en los asuntos relativos a las políticas públicas, dado que las notas para la participación ciudadana a nivel nacional resultó ser de 3.83, a nivel regional 3.38, y a nivel local 3.44. Por tanto, pareciera ser que el sistema está dominado por una estructura cerrada en donde todo lo que se refiere a las políticas públicas se conceptualiza, se desarrolla y se implementa sin una apertura a la sociedad civil. En consecuencia, los proyectos que son implementados no tienen necesariamente una aprobación popular. Abordar los problemas solamente desde la alta dirección puede ocasionar falta de eficacia en la implementación. Tampoco existe la ley de transparencia de los municipios.

Al igual que en el caso de la gestión, la percepción que los encuestados tienen del sistema de planificación no es buena junto con el funcionamiento del sistema, lo que puede deberse a la percepción de ineficiencia del ministerio en el desarrollo y coordinación de actividades para alcanzar objetivos de desarrollo, particularmente desde las dos últimas décadas.

\section{Conclusiones y recomendacio- nes}

\section{Conclusión}

Comparativamente al modelo estructural teórico, el sistema de planificación de Haití está bastante bien diseñado, ya que cuenta con casi todos los miembros que requiere el esqueleto de un sistema de planificación, pudiendo entregar bienes y servicios a la población. Al respecto, los cuellos de botellas que habíamos identificado en la estructura corresponden, particularmente, a la falta de una Dirección de Desarrollo Institucional dentro de la estructura de las direcciones, la falta de una Dirección de Modernización del Estado dentro de los ámbitos de acciones, la falta de un plan de desarrollo institucional y un plan de desarrollo territorial dentro de los instrumentos y la falta de una comisión de política fiscal y de los dispositivos de enlace institucional dentro de los mecanismos de articulación.

A pesar de que existe un sesgo en el sistema de planificación de Haití hacia el subsistema de la inversión pública, no existe una ley de la inversión pública que reglamente los proyectos de los distintos actores. Esta ley sería muy importante en la medida en que pueda reglamentar los proyectos y sus financiamientos y, a su vez, aplicar sanciones.

No existe una ley de ordenamiento del territorio. A pesar de que la Constitución de la República de Haití de 1987 y su versión enmendada reconoce la descentralización como un pilar muy importante para un desarrollo equilibrado, todavía no existe una ley de descentralización que establezca los criterios generales del proceso mismo, que regule la formación de las regiones y las relaciones del gobierno con en sus distintos niveles.

Falta también una ley orgánica de las municipalidades en Haití, pudiendo normar la naturaleza, finalidad, competencia, funciones, organización, recursos, patrimonio, relaciones e instituciones de apoyo de las municipalidades del país.

Tampoco existe una ley de transparencia, que reconozca y regule los derechos individuales al acceso a la información de las instituciones, organismos del Estado y la sociedad. Esta ley recoge, en muchos países latinoamericanos, la necesidad de garantizar la participación democrática de los ciudadanos mediante el acceso completo a la información sobre los asuntos del Estado, que garantiza el derecho de información con el objetivo primero de establecer un modelo administrativo abierto y democrático, eficiente administrativamente a través de rendición 
de cuentas y supervisión ciudadana.

Existe un desequilibrio en el funcionamiento de las direcciones y de las unidades del Ministerio de Planificación de Haití, lo que puede resultar de una falta de capacitación o de una inadecuada repartición de los recursos humanos. Es un problema de gestión que debe ser solucionado a nivel estratégico en la medida en que las distintas estructuras directivas del ministerio no funcionan de manera hermética, sino que se relacionan entre ellas.

A nivel estratégico, el Ministerio de Planificación funciona mucho mejor que a nivel programático y operacional. Esto constituye un nodo crítico de gestión, que corresponde a una estructura burocrática en la que la toma de decisiones está fuertemente concentrada en los altos niveles de administración.

Del mismo modo, la buena relación que mantiene el ministerio en el ámbito de la cooperación externa en cuanto a los otros ámbitos constituye un nodo crítico de gestión por el hecho de que, en la práctica, eso se traduce en una dependencia muy fuerte a la cooperación internacional. Cabe destacar que la proporción de la ayuda externa en el presupuesto nacional supera el $50 \%$ en los últimos años. Esto es un problema muy relevante cuando consideramos un detenimiento del financiamiento externo o el peso político que puede jugar la presión internacional sobre la política interna.

Paradójicamente, a pesar de que el Ministerio cuenta con un Centro de Técnicas de Planificación y de Economía Aplicada (CTPEA) y un Servicio de Becas en la Dirección de Cooperación Externa, algunos piensan que el ministerio no ha desarrollado una política pública orientada a capacitar eficazmente a sus empleados. Al respecto, puede que no exista un problema de capacitación en sí, pero lo que podría existir es un problema de gestión de estos capacitados, ya que se observa una fuga cada vez más de los empleados del ministerio hacia otras instituciones con mejores remuneraciones.

Todavía no se han implementado completamente los mecanismos para la participación ciudadana, lo que es un tema muy importante hoy en día en las políticas públicas ya que permite empoderar a los ciudadanos que son los beneficiarios de los proyectos que se implementan. Asimismo, a nivel micro, esto permitiría aterrizar muy eficientemente los proyectos de desarrollo, dado que los expertos de la administración tienen en la mayoría de los casos una visión global y a veces no detallada en cuanto a la realidad que está viviendo la población. Otra ventaja que tiene este mecanismo de participación es de impulsar el desarrollo local y la democracia participativa a través de la integración de la comu- nidad al ejercicio de la política pública.

\section{Recomendación}

La legislación de una ley orgánica de planificación dentro de la Constitución permitiría establecer los grandes lineamientos para la organización y funcionamiento de un sistema de planificación orientado al logro de los objetivos estratégicos del desarrollo social de la nación, ejerciendo una planificación que permita administrar los recursos eficientemente, con el fin de lograr una justa distribución de la riqueza. Además, constituirá un marco legal para todos los actores interviniendo en el sistema.

Es importante integrar al Ministerio de Planificación una Dirección de Desarrollo Institucional o de fortalecimiento institucional que sea capaz de cubrir las debilidades del sistema en materias institucionales, reforzando la capacidad, el desarrollo metodológico, análisis, diseño e implementación de proceso. Es necesario evaluar si es conveniente que la Dirección de Modernización del Estado siga la estructura de la Primature, o si debiera integrarse al Ministerio de Planificación, dándole una orientación enfocada al desarrollo económico y social.

Creación de una metodología o marco normativo para la elaboración y la regulación de los planes, que mejore el proceso de formulación, concertación y adopción del plan de Desarrollo.

Legislar sobre la inversión pública con el fin de obligar a los actores a coordinar esfuerzos para la implementación de los proyectos de desarrollo y fortalecer la Dirección de la Cooperación Externa, jugando un rol mucho más importante en cuanto a los proyectos de desarrollo a financiamiento externo. Del mismo modo es necesario crear una ley de ordenamiento del territorio y fortalecer la Dirección del Ordenamiento del Territorio que fiscalice el diseño e implementación de los planes territoriales.

Creación de una ley de descentralización que establezca la finalidad, principios, objetivos, y criterios generales del proceso de descentralización; que regula la formación de las regiones; fije las competencias de los tres niveles de gobierno; determine los bienes y recursos de los gobiernos regionales y locales; y por ultimo regule las relaciones de gobierno en sus distintos niveles. Al respecto, se debería transferir funciones, atribuciones, responsabilidades y recursos de la Administración Central hacia los departamentos a efectos de distribuir los recursos y los servicios de acuerdo con las necesidades de las respectivas circunscripciones territoriales.

También sería necesaria de crear una ley de Mu- 
nicipalidad pudiendo normar la naturaleza, finalidad, competencia, funciones, organización, recursos, patrimonio, relaciones e instituciones de apoyo de las municipalidades del país.

Creación de una ley de transparencia y de acceso a la información que tenga como finalidad promover la transparencia de los actos del Estado y regular el derecho fundamental de los ciudadanos a la información, con el fin de reforzar la fiscalización ciudadana evitando abusos de poder y actos de corrupción.

También se hace necesario nivelar la eficacia de todas las Direcciones y las Unidades; para ello, sería importante reforzar estas unidades en recursos humanos capacitados y en métodos de gestión con el fin de tener un equilibrio entre las distintas estructuras de la planificación.

Se sugiere un reforzamiento del Ministerio de Planificación en los niveles programáticos y operacionales y una desconcentración en el proceso de toma de decisiones. Esto permitiría flexibilizar y responder mejor a las innovaciones y a los cambios de forma positiva y proactiva. Al respecto, es necesario que el ministerio mejore su relación con los ejes de coordinación sectorial, territorial y con estructuras de planificación externa y además ponga en práctica mecanismos de desconcentración y de descentralización para que el sistema de planificación atienda en forma constante y detallada al desenvolvimiento de los programas a nivel regional, local y sectorial. Además, fortalecer y mejorar la coordinación sectorial por parte de los directivos y profesionales sectoriales del ministerio.

El ministerio debe apoyarse sobre la declaración de parís de 2005 y el programa de acción de Dakar para hacer más eficaz la ayuda externa y responsabilizar a los países socios de los compromisos de esta declaración. Se debe trabajar para reducir el papel de la cooperación internacional a nivel nacional o disminuir la tasa de financiamiento externo de los proyectos para el desarrollo.

Para solucionar, la correlación que tiene la ejecución de los planes con la coyuntura política, se recomienda generar una cultura de rendición de cuenta, evaluación de los planes y un marco de seguimiento de los planes con cuenta pública.

En el mismo sentido se recomienda que el Mi- nisterio refuerce los distintos instrumentos de planificación sobre todo a nivel programático y operacional con el fin de repartir las acciones racionalizadoras y previsoras de los gobiernos a todos los niveles.

Para mejorar la eficacidad del ministerio, sería importante que el Ministerio desarrolle una política de capacitación eficaz para reforzar todas las estructuras del Ministerio de Planificación.

\section{Referencias}

Barrantes, R. (2002). Investigación: Un Camino al Conocimiento, un Enfoque Cuantitativo y Cualitativo. Editorial Universidad Estatal a Distancia, San José'.

Crozier, M. (1969). El fenó'meno burocrático. Amorrortu, Buenos Aires.

Giddens, A. (1994). Consecuencia de la Modernidad. Alianza Editorial, Madrid.

Katz, D. y Kahn, D. (1977). Psicología social de las Organizaciones. Trillas, México D.F.

Lahera, E. (2008). Introducció'n a las Políticas Pú'blicas. Fondo de Cultura Econó'mica, Santiago de Chile.

Mintzberg, H. (1999). La Estructuración de las Organizaciones. Ariel, Barcelona.

Moore, M. (1998). Gestión estratégica y creació'n de valor en el sector público. Paidos, Barcelona.

Motta, P. R. (1993). La Ciencia y el Arte de ser dirigente: un instrumento indispensable para el desarrollo personal y profesional de actuales y futuros dirigentes. Editores Tercer Mundo, Bogotá.

Pacheco, J. (2012). Gestión del Cambio Social desde la Administración $n$ Pública y la Planificación en América Latina. ILPES, Santiago de Chile.

Saavedra Guzman, R. (2003). Planificació'n del Desarrollo. Fundaciónn Universidad de Bogotá Jorge Tadeo Lozano, Bogotá.

Weber, M. (1964). Economía y Sociedad: esbozo de sociología comprensiva. Fondo de Cultura Econó'mica, México D.F. 